\title{
Temporomandibular Disorders: Fundamental Questions and Answers
}

\author{
Frantzeska Karkazi (D), Fulya Özdemir (iD
}

Department of Orthodontics, Marmara University, Istanbul, Turkey

Cite this article as: Karkazi F, Özdemir F. Temporomandibular Disorders: Fundamental Questions and Answers. Turk J Orthod 2020; 33(4): 246-52.

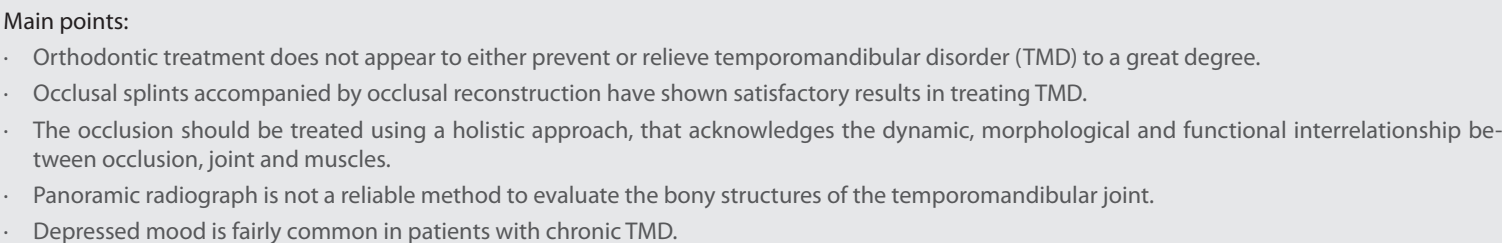

\begin{abstract}
This review aimed to present the current evidence-based answers to a questionnaire which was developed to evaluate the beliefs and knowledge of dental professionals concerning temporomandibular disorder (TMD). A literature review was conducted using the PubMed/Medical Literature Analysis and Retrieval System Online (MEDLINE), Cochrane, Google Scholar, and Scopus search engines from January 1980 to June 2020 corresponding to TMD diagnosis, causes, management, and consequences. A total of 50 articles were considered relevant and selected for full review. The etiology of TMD is multifactorial and complicated and involves several direct and indirect factors. Orthodontic treatment does not appear to either prevent or relieve TMD to a great degree. Nevertheless, condylar and occlusal stability reduces the risk of TMD development. As a result, an assessment of the function of masticatory system prior to beginning orthodontic therapy is fundamental. If signs and symptoms of TMD are significantly present, they should be managed before any orthodontic intervention. Moreover, psychological disorders are strongly associated with joint and facial pain. Thus, professionals who work with chronic TMD patients need to be aware of the psychological aspects of chronic pain and refer the patient for psychological evaluation when warranted. Future research elucidating a cause-effect relationship and neurobehavioral processes underlining chronic pain should be performed.
\end{abstract}

Keywords: Diagnosis, etiology, questionnaire, temporomandibular disorder, treatment

\section{INTRODUCTION}

Temporomandibular disorder (TMD) is a broad term referring to all neuromuscular and musculoskeletal conditions of the masticatory muscles, temporomandibular joint (TMJ), and the adjacent structures (1). TMD is described as the most common orofacial pathology of non-dental origin, and it affects mostly women and those aged 20-45 years (2). The most frequent clinical features are chronic myofascial pain, masticatory muscle pain, and limited range of mouth opening (3). However, other symptoms, such as earache, headache, neuralgia, and toothache, may also be present (4). Although the etiology of TMD is complicated, it is likely multifactorial with biomechanical, neuromuscular, psychosocial, and biological influences. Plausible causes involve trauma, functional shift, parafunctional habits, occlusal overloading, increased joint friction, depression, stress, anxiety, and alexithymia (5). The role of each of these potential components is still controversial, and they can contribute either independently or collectively (6). TMD treatment is classified as non-invasive, mini-invasive, and invasive. Although each of these treatment modalities provides favorable results in reducing TMJ pain, no specific therapy 
has been shown to be predominantly effective over the other (7). Moreover, one determinant of successful TMD treatment, which is frequently overlooked, is the practitioner's knowledge and beliefs concerning the disorder itself. Thus, patients are often misdiagnosed and undergo various therapies for unrelated disorders; a fact that often leads to frustration, dissatisfaction, and compromised quality of life (8).

In 1993, Le Resche, Truelove, and Dworkin (9) developed a survey to evaluate the knowledge and beliefs of general dentists. The questionnaire included 4 fields of study: pathophysiology, chronic pain, psychophysiology, and psychiatric disorders. Items were answered on an 11-point scale, ranging from 0 "strongly disagree" to 10 "strongly agree." Since then, multiple scientific articles have used the questionnaire for surveying either general dentists and/or orthodontists $(10,11)$. In 2016, the questionnaire was modified by Porto et al. (12) to meet the current diagnostic and treatment standards. The aim was to evaluate the changes in experts' knowledge and beliefs about TMD after the administration of the first such survey by Le Resche, Truelove, and Dworkin in 1993. The results showed that the knowledge and beliefs related to TMD have not significantly changed in the past 20 years. Thereafter, the specific updated survey has been used again to assess the awareness of both general dentists and orthodontists about TMD (13).

This study aimed to answer the 38 questions of the questionnaire of Porto et al. (12) as an attempt to update the knowledge of dental professionals, as well as, to present the current evidence-based knowledge concerning the etiology, diagnosis, consequences, and management of TMD. A literature review was conducted using the PubMed, Medical Literature Analysis and Retrieval System Online (MEDLINE), Cochrane, Google Scholar, and Scopus search engines from January 1980 to June 2020 corresponding to TMD diagnosis, causes, management, and consequences. A total of 50 articles were considered relevant and selected for full review.

\section{CLINICAL AND RESEARCH CONSEQUENCES}

In this study, the questionnaire of Porto et al. (12) was used as a reference point to answer controversial issues of TMD in the following 4 domains: pathophysiology (Table 1), chronic pain (Table 2), psychophysiology (Table 3), and psychiatric disorders (Table 4).

\section{Pathophysiology Domain of Temporomandibular Disorder}

\section{Occlusal equilibration}

Occlusal equilibration involves selective iatrogenic grinding of dental surfaces to increase intercuspation in the centric relationship or to enhance guidance during excursions. Treatment objectives include the establishment of an acceptable centric relation with a harmonious intercuspal position and an acceptable lateral and protrusive guidance. However, clinicians are recommended to avoid this method as an initial TMD therapy in the absence of restorative prematurities, because it is not confirmed by any solid biological background or analogy with other musculoskeletal
Table 1. Pathophysiology domain and the most highly rated answers among orthodontists in the questionnaire of Porto et al. (12)

\section{Statement}

Score (\%)

Occlusal equilibration is a useful early treatment for TMD.

50.4 disagree

Orthodontic treatment can prevent the onset of TMD.

Arthroscopic surgery is almost completely effective in repositioning the disc in patients with internal derangements.

59.8 disagree

Orthodontic therapy is the best treatment to resolve TMD in a patient with a skeletal malocclusion.

58.1 disagree

67.5 disagree

TMD caused by trauma is much more difficult to treat and has a far worse prognosis than other types of TMD.

Panoramic film is a reasonable method to evaluate the bony structures of the TMJ.

33.1 agree

66.7 disagree

When bony changes are seen on a panoramic film, a tomogram is mandatory to define the treatment plan.

45.7 agree

The presence of arthritic changes on tomograms, along with crepitus in the joint, indicates the need for treatment.

48.5 disagree

The position of the condyle in the fossa as seen on the tomogram is a very accurate indicator of internal derangement.

53.8 disagree

Mandibular repositioning splints are more effective than maxillary repositioning splints.

47.5 disagree

Splint therapy is only effective when the splint is used for more than 16 hours/day.

40.8 disagree

Nocturnal bruxism is caused by occlusal interference. 71.2 disagree

Ice packs and/or heat packs and passive muscle stretching are good early treatments for TMD.

74.7 agree

All individuals with clicking TMJs require treatment.

Balancing interference is commonly related to TMD.

96.2 disagree

34.5 agree

TMD: Temporomandibular disorder; TMJ: Temporomandibular joint

Table 2. Chronic pain domain and the most highly rated answers among orthodontists in the survey of Porto et al. (12)

Statement

Score (\%)

PRN narcotics ("as needed" for pain) are a treatment of choice when TMD pain is severe.

55.3 disagree

Antidepressants are never indicated in the management of TMD.

60.1 disagree

An extensive history of previous treatment failures in a patient with TMD is usually an indication for surgery.

Chronic pain is a behavioral as well as a physical problem.

55.7 disagree

63.4 agree

Although some patients with TMD have psychological problems, these problems are usually unrelated to their pain.

70.8 disagree

Poor quality of sleep is a major factor in the development of TMD.

21.1 agree

Difficulty with sleep is a common finding in chronic pain.

Some patients use pain as an excuse to avoid unpleasant chores.

72.2 agree

41 agree

Behavior modification treatments are appropriate for patients with chronic TMD pain.

69.4 agree

Patients with chronic TMD should be advised to rest and limit their work and social activities when they are experiencing pain.

34.1 disagree 
Table 3. Psychophysiology domain and the most highly rated answers among orthodontists

\begin{tabular}{|lc|}
\hline Statement & Score (\%) \\
\hline $\begin{array}{l}\text { The mechanisms of acute and chronic pain are } \\
\text { the same. }\end{array}$ & $\begin{array}{c}82.6 \text { disagree } \\
\text { Biofeedback can be useful for treating TMD. }\end{array}$ \\
$\begin{array}{l}\text { Oral parafunction habits are often significant in the } \\
\text { development of TMD. }\end{array}$ & 71 agree \\
$\begin{array}{l}\text { Patients with TMD who clench/brux do so either } \\
\text { during the day or at night, but not both. }\end{array}$ & 78.6 disagree \\
$\begin{array}{l}\text { Stress management is indicated in many patients } \\
\text { with TMD. }\end{array}$ & 89.8 agree \\
$\begin{array}{l}\text { Stress is a major factor in the development of TMD. } \\
\text { Tension and stress increase jaw muscle EMG levels }\end{array}$ & 78.3 agree \\
in susceptible patients. & 79.6 agree \\
$\begin{array}{l}\text { Progressive muscle relaxation is not an effective } \\
\text { treatment for TMD. }\end{array}$ & 61 disagree \\
$\begin{array}{l}\text { Information on the daily pattern of the TMD } \\
\text { symptoms can be helpful for identifying } \\
\text { contributing factors. }\end{array}$ & \\
\hline TMD: Temporomandibular disorder; EMG: Electromyography & \\
\hline
\end{tabular}

Table 4. Psychiatric domain and the most highly rated answers among orthodontists

\section{Statement}

Score (\%)

Clinical depression is rare in patients with chronic TMD. 65.2 disagree

Depressed mood is fairly common in patients with chronic TMD.

70.1 agree

Anxiety disorders are more common in patients with TMD than in the population at large.

49.7 agree

Depression can be an important etiologic factor in chronic pain.

73.5 agree

TMD: Temporomandibular disorder

areas. The two major drawbacks are the irreversibility of the technique and the possible development of tooth sensitivity (14).

\section{Cryotherapy}

Cryotherapy includes the application of the local use of low temperatures in a traumatic and/or inflammatory region. Local effects involve vasoconstriction and reduction of inflammation, pain, and muscle spasms. Nonetheless, scientific data proving its efficacy are insufficient, probably owing to the introduction of more updated techniques, such as ultrasound and transcutaneous electrical stimulation (15).

\section{Orthodontics and Temporomandibular Disorder}

Whether abnormal skeletal characteristics cause TMD or vice versa has not been clearly clarified yet in the literature. Although, TMD has been associated with posterior crossbite, anterior open bite, Angle Class II and III malocclusions, and increased overjet (16). Some acute malocclusions (sudden occlusal changes) might also arise as a consequence of a joint or muscle disorder. Even though, recent studies have concluded that orthodontic treatment cannot prevent TMD to a great degree. Since, the difference of TMD incidents between orthodontically treated and untreated subjects was not statistically significant $(2,17,18)$. Orthodontists should recognize how occlusion can become a risk factor for TMD and incorporate an orthopedic approach in cases of TMD symptomatology to establish both condylar and occlusal stability. In more detail, occlusal splints accompanied by occlusal reconstruction have shown satisfactory results in treating TMD (19-21). Imai et al. (21) concluded that $60 \%$ of patients achieved pain relief following the aforementioned approach. Moreover, TMD symptoms are not likely to reoccur following the establishment of balanced occlusion and proper alignment after orthodontic treatment.

\section{The Role of Panoramic Radiography in the Temporomandibular Disorder Diagnosis}

Although panoramic radiography is the most common initial diagnostic tool in dentistry; it fails to exclude significant morphological abnormalities in TMJ. More specifically, due to the superimposition of the skull base and the zygomatic arch, only excessive alterations in the articular tubercle can be observed. Therefore, bony changes, such as erosions and osteophytes, are difficult to be detected in conventional radiographs. Cone-beam computed tomography (CBCT) is considered superior to $2 \mathrm{D}$ radiography in illustrating the condylar head morphology and evaluating the presence of condylar deformities, such as osteoarthrosis. On the other hand, magnetic resonance imaging (MRI) is the recommended imaging modality for the evaluation of the disc-condyle relationship, the soft tissues, and for the diagnosis of the disc displacement (22). As a result, the use of fused MRI and CBCT images to visualize the TMJ in a single display can significantly improve the examiners' reliability and accuracy of assessment of disc positions.

\section{Temporomandibular Osteoarthritis}

When osteoarthritic changes are observed in 2D radiographs, an extended and detailed inspection of the patient's medical history and a scrutinous physical examination should be performed as well. Clinical features indicating TMD include joint tenderness, crepitus, and pain during mouth opening and lateral movements. The information gathered should be integrated with CBCT imaging to exclude any differential diagnosis. Radiographically, the disease includes cortical bone erosion and degenerative bone changes, such as sclerosis, flattening, subchondral cysts and osteophytes. The abovementioned signs of temporomandibular osteoarthritis (OA) correspond to different disease stages. Erosive lesions and reduction in joint space indicate acute or early stages; whereas sclerosis, flattening, subchondral cyst, and osteophytes illustrate the later stages of OA (23). However, even when the aforementioned radiographic changes are observed, TMD treatment is indicated only if both symptomatology and considerably limited function are present.

\section{Occlusal Splint Therapy}

Dental occlusal splints have been the mainstay of TMD treatment over the past decades in cases of discopathies and inflammatory/degenerative changes. The advantages of occlusal splints include occlusal stability, balance in centric relation, as well as reduction of tension and pain in the joint and the adjacent tissues. The opinion that splints must be used for at 
least 16 hours/day to be effective has not been proved yet in the literature (24). Thus, a prospective study could clarify the relationship between the hours of application of occlusal splints and their effectiveness in establishing condylar stability. Moreover, clinical comparisons between the maxillary and mandibular occlusal splints have shown no significant differences in their treatment outcomes. More specifically, maxillary and mandibular splints seem to be equally beneficial in reducing TMD signs and symptoms. The maxillary appliance provides a better stabilization than the mandibular appliance because all of the mandibular teeth can contact against a flat surface. The choice of maxillary or mandibular splint depends mostly on clinical observations. As a case in point, fabricating the appliance on the arch with missing teeth increases the intercuspal contacts and thus equilibrates the occlusion. Furthermore, in patients who are expected to benefit from daytime application, a mandibular splint, which is rather less noticeable and produces fewer speech problems, is more befitting. While, in cases of severe overjet, the construction of anterior and lateral canine guidance is easier established with maxillary stabilization splint. If the appliance is to be worn only during sleep, the majority of dentists choose maxillary occlusal splints, although this choice is considered more like a pattern of tradition (25).

\section{Clicking of the Temporomandibular Joint}

Clicking occurs when the condylar head extends over the edge of the displaced articular disc during mouth opening and/or closing. Thus, clicking alone is not an indication for TMD treatment. However, when clicking sounds are noted, a detailed clinical evaluation should be performed. This includes evaluation of the occlusion, detection of occlusal interferences, joint palpation, and assessment of the masticatory muscles (26).

\section{Arthroscopy}

TMJ arthroscopy serves as either a diagnostic procedure or a minimally invasive TMD treatment for internal derangement with arthroscopic lysis or lavage. Joint visualization ensures accurate stage diagnosis and identification of OA. Moreover, arthroscopy includes fluid infusion under pressure to expand the TMJ and to break any adherences that are liable for reduced condyle movements. The success rate in internal derangement cases is high in terms of pain relief and maximum mouth opening. In a recent systematic review, arthroscopy lysis and lavage were found to have superior efficacy in functional outcome and degree of pain control than arthrocentesis (27).

\section{Balancing Interference}

The relation between occlusal interference and TMD shows no consensus in the literature yet. Though, it has been proven that occlusal interferences might cause minor transient changes in the myoelectric contraction patterns of the jaw muscles and short-term clinical symptoms and signs, such as pain and fatigue of the jaw muscles, headache and clicking (28). Other studies support the individual differences in vulnerability to occlusal interference, such as a previous history of TMD or even psychological factors. Therefore, further research and more updated methods are required (29).

\section{Trauma Cases and Temporomandibular Disorder}

Trauma can be divided into macrotrauma (for example, whiplash-type injury) and microtrauma (for example, parafunctional habits). Traumatic microlesions of the masticatory muscle fibers release local inflammatory mediators, such as bradykinin, prostaglandins, substance $P$, and histamine. These substances can transmit nociceptive impulses to the central nervous center, triggering both peripheral and central sensitization. Trauma is defined as a predisposing or initiating cause of TMD. A study including 400 patients with TMD revealed that TMJ pain was directly related to a positive history of whiplash trauma in $24.5 \%$ of the patients (30). However, there are no scientific data to confirm that treating patients with TMD with a trauma history is a more arduous task than treating patients with TMD with differential diagnosis (14).

\section{Chronic Pain Domain of Temporomandibular Disorder}

\section{Medical Treatment of Temporomandibular Disorder}

Pharmaceutical treatment of TMD includes non-opioid analgesics (acetaminophen), mild opiates (tramadol hydrochloride), anti-inflammatory drugs (ibuprofen and diclofenac), and/or muscle relaxants (tetrazepam).

Pro re nata (PRN) narcotics can be prescribed to alleviate acute pain symptoms when non-steroidal anti-inflammatory drugs are contraindicated. In any case, both careful selection of patients and prescription at regular intervals for a specific period (for example, 3 times a day for 10 days) are mandatory. Owing to the significant reduction in intracapsular pain, opioids may also be injected intra-articularly. List et al. (31) concluded that intra-articular morphine increases the pain threshold of the affected joint. Narcotics prescribed to alleviate chronic pain associated with TMD should be eschewed due to the increased risk of abuse, tolerance, and addiction.

Antidepressants can alleviate TMD pain because of their analgesic impact on chronic pain, independently of their antidepressant effect. Tricyclic antidepressants (TCAs) appear to be the most efficient in controlling chronic pain; $25 \mathrm{mg} /$ day of amitriptyline is adequate in reducing pain and discomfort in patients with TMD (32). However, it has been suggested that the effects of amitriptyline are equal to those illustrated in the placebo groups. Thus, the reported benefits of amitriptyline are plausibly based on the difficulty most patients have in measuring subjective sensations of pain and discomfort. Moreover, typical side effects include xerostomia, sedation, memory impairment, constipation, and orthostatic hypotension (33).

Other antidepressants, which are frequently prescribed to patients with TMD, are selective serotonin reuptake inhibitor antidepressants (SSRIs). These medications provide greater compliance rates owing to the reduced incidence of side effects than TCAs. Moreover, SSRIs result in fewer antihistaminergic, anticholinergic, and antiadrenergic incidences. However, gastrointestinal disturbances, headache, sexual dysfunction, dry mouth, and excessive sweating are some of the adverse drug reactions (34). 
Indications of Invasive Temporomandibular Disorder Treatment Absolute indications of surgical interventions include ankylosis (fibrous or osseous joint fusion), neoplasia (osteochondroma), developmental disorders (condylar hyperplasia), and dislocation (chronic or recurrent), whereas relative indications are internal derangement, arthritic conditions, and trauma (35).

\section{Psychological Disorders and Temporomandibular Disorder}

TMD is a somatic and psychological condition involving fatigue, sleep disturbances, anxiety, and depression. Psychological problems (e.g. depression and anxiety) may cause stress to the TMJ and masticatory muscles via the stimulation of the sympathetic nervous system (36). In addition, current research has confirmed the relationship between psychological disorders/dysfunctions and chronic pain (37). At this point, it should be noted that patients with a high level of psychological distress benefit from multimodal treatment approaches (38).

\section{Sleep Quality in Patients with Temporomandibular Disorder} The relationship between pain and sleep quality in patients with TMD is well documented. Patients with TMD show a high prevalence of sleep disorders. Nevertheless, whether TMD is the cause rather than the result of sleep disorders has not been clarified yet in the literature (39). According to a recent systematic review, patients with TMD have two or more sleep disorders in a percentage of $43 \%$, with insomnia (36\%) and sleep apnea (28.4\%) as the most frequent (40).

\section{Behavioral modification}

Although, the effectiveness of behavioral modifications in chronic pain management is unable to be demonstrated scientifically, owing to the lack of a standard treatment methodology; in general, the combination of cognitive-behavioral treatment concentrating on somatization, readiness, and self-efficacy provides beneficial outcomes in managing chronic TMD pain (41).

Moreover, despite the consequences of TMD pain on physical and psychological health, practitioners should encourage their patients to maintain an adequate activity level and correct posture, abstain from excessive rest, and perform various chores when physically feasible.

\section{Psychophysiology Domain of Temporomandibular Disorder}

\section{Pain mechanisms}

The mechanisms of acute and chronic pain differ greatly from one another. The alteration from acute to chronic pain occurs in a pathophysiological and histopathological manner. The stimuli initiating a nociceptive response vary; however, the mechanism of interaction between the receptors and the peripheral internal defense system is similar (42).

\section{Biofeedback}

As mentioned earlier, psychological factors, such as stress, mental tension, anxiety, or depression, may be involved in TMD. To improve the effectiveness of TMD treatment, patients should overcome their stress and other TMD-associated psychological factors via bio-behavioral treatment and other treatment ap- proaches, such as therapeutic education, cognitive-behavioral therapy, and methods of physiological self-regulation.

Biofeedback regulates muscle tension and decreases muscle pain. This method provides extrinsic feedback that otherwise would be unknown to the recipient. Thus, patients acquire supplementary information in addition to the intrinsic feedback, which is naturally acquired.

Electromyography (EMG) biofeedback is the technique of getting feedback from the body to measure the frequency, intensity and duration of muscle spasms. It can be used to either enhance the activity of weak muscles or to attenuate the tone of muscle spasms (43). Shedden Mora et al. (44) have found that patients undergoing biofeedback-based cognitive-behavioral therapy showed greater improvement in pain coping skills and increased satisfaction than those following an occlusion-centered approach.

\section{Parafunctional habits}

Parafunctional functions are one of the main evidence-based causes of TMD. Oral parafunctions include bruxism, clenching, excessive gum chewing, lip/nail biting, and non-nutritive sucking. A recent study has shown that excessive gum chewing (more than 4 hours/day) is positively correlated with auricular pain and either clicking or clenching. It should also be noted that $87.5 \%$ of individuals with disc displacement and TMD pain are bruxers (14).

\section{Electromyography Levels of Patients with Temporomandibular Disorder}

It has been proven that stress and tension are associated with increased EMG levels in the jaw muscle. Tsai et al. (45) concluded that the EMG activity of the masticatory muscles was increased significantly under conditions of induced stress. Therefore, progressive muscle relaxation is considered an effective therapeutic method for TMD to increase the range of motion and decrease pain.

Identifying the factors contributing to TMD and educating the patients to relieve stress is one of the most challenging aspects. Preferably, a daily symptom pattern should be used as a guide. However, when the identification of the daytime contributing factors cannot be accomplished, patients should record the incidents of pain, clenching, and masticatory muscle tension together with the associated events on an hourly basis. This often helps the patients to identify which events are mostly related to their pain. Patients may require additional psychological support in ways to relax and/or break habits (46).

\section{Psychiatric Domain of Temporomandibular Disorder}

\section{Depression}

TMD is strongly associated with elevated levels of depression and anxiety, and women are more susceptible to severe depression. Women show an increased level of monoamineoxidase, an enzyme that metabolizes norepinephrine, serotonin, and dopamine, owing to premenstrual and postmenstrual hormonal changes (47). However, depression is also associated with articular pain (48). 


\section{Anxiety}

The link between chronic pain and mental balance seems to have a bidirectional etiology, with pain-causing fluctuations in mental balance and vice versa. The level of anxiety is directly associated with muscle hyperactivity and facial pain. Nevertheless, anxiety shows no significant differentiation between the sexes (49). Moreover, conditions such as generalized anxiety disorders and social anxiety disorder increase the risk of developing TMD (50).

\section{CONCLUSION}

TMD is the most frequent type of non-odontogenic orofacial pain, which often results in compromised welfare of individuals with TMD. As a result, it has been the stimulus for a plethora of research investigations. However, despite the plethora of studies, there is a lack of comprehensive, unified, and systematic characterization of the etiology, diagnosis, and treatment of TMD. On the positive side, a consensus has been reached on four points: First, orthodontic treatment does not appear to either prevent or relieve TMD to a great extent. Nevertheless, condylar and occlusal stability reduces the risk of TMD development. Second, panoramic X-rays do not show valid morphological changes in the TMJ. Third, PRN narcotics and antidepressants can both be prescribed for pain relief to TMD patients. Psychological disorders, such as depression and anxiety, are strongly associated with joint and facial pain. Finally, yet importantly, current evidence supports the positive effect of biofeedback-based cognitive-behavioral therapy on the pain coping skills of individuals with TMD. Future research elucidating the cause-effect relationship and neurobehavioral processes underlining chronic pain is warranted.

Peer-review: Externally peer-reviewed.

Author Contributions: Supervision - F.Ö.; Design - F.Ö.; Data Collection and/or Processing - F.K.; Analysis and/or Interpretation - F.Ö., F.K.; Literature Search - F.Ö., F.K.; Writing Manuscript - F.K.; Critical Review - F.Ö. F.K.

Conflict of Interest: The authors have no conflict of interest to declare.

Financial Disclosure: The authors declared that this study has received no financial support.

\section{REFERENCES}

1. Gauer RL, Semidey MJ. Diagnosis and Treatment of temporomandibular disorders. Am Fam Physician 2015; 91: 378-86.

2. Špalj S, Šlaj M, Athanasiou AE, Žak I, Šimunović M, Šlaj M. Temporomandibular disorders and orthodontic treatment need in orthodontically untreated children and adolescents. Coll Antropol 2015; 39: 151-8.

3. Miettinen O, Lahti S, Sipilä K. Psychosocial aspects of temporomandibular disorders and oral health-related quality-of-life. Acta Odontol Scand 2012; 70: 331-36. [Crossref]

4. Gnauck M, Magnusson T, Ekberg E. Knowledge and competence in temporomandibular disorders among Swedish general dental practitioners and dental hygienists. Acta Odontol Scand 2017; 75: 429-36. [Crossref]

5. Tanaka E, Detamore MS, Mercuri LG. Degenerative disorders of the temporomandibular joint: etiology, diagnosis, and treatment. J Dent Res 2008; 87: 296-307. [Crossref]

6. Ahlberg J, Nikkila $H$, Kononen $M$, Partinen $M$, Lindholm H, Sarna $\mathrm{S}$, et al. Associations of perceived pain and painless TMD-related symptoms with alexithymia and depressive mood in media personnel with or without irregular shift work. Acta Odontol Scand 2004; 62: 119-23. [Crossref]

7. Wieckiewicz M, Boening K, Wiland P, Shiau YY, Paradowska-Stolarz A. Reported concepts for the treatment modalities and pain management of temporomandibular disorders. J Headache Pain 2015; 16: 106. [Crossref]

8. Manfredini D, Guarda-Nardini L, Winocur E, Piccotti F, Ahlberg J, Lobbezoo F. Research diagnostic criteria for temporomandibular disorders: A systematic review of axis I epidemiologic findings. Oral Surg Oral Med Oral Pathol Oral Radiol Endod 2011; 112: 453-62. [Crossref]

9. Le Resche L, Truelove EL, Dworkin SF. Temporomandibular disorders: a survey of dentists' knowledge and beliefs. J Am Dent Assoc 1993; 124: 90-4, 97-106. [Crossref]

10. Coêlho TG, Caracas HC. Perception of the relationship between TMD and orthodontic treatment among orthodontists. Dental Press J Orthod 2015; 20: 45-51. [Crossref]

11. Espinosa IA, Pérez EM, Gonzalez YM, Corona A. Assessment of knowledge on temporomandibular disorders among Mexican dental educators. Acta Odontol Latinoam 2016; 29: 206-13.

12. Porto F B, Litt M, Jennings M E, Rifaey H, Reisine S. Knowledge and Beliefs Regarding TMD: Has Anything Changed After 20 Years? Health Scope 2016; 5: 313-28. [Crossref]

13. Porto F, Harrell R, Fulcher R, Gonzales T. Knowledge and beliefs regarding temporomandibular disorders among orthodontists. Am J Orthod Dentofacial Orthop 2019; 156: 475-84. [Crossref]

14. Keshvad A, Winstanley RB. Comparison of the replicability of routinely used centric relation registration techniques. J Prosthodont 2003; 12: 90-101. [Crossref]

15. Fedorczyk J. The role of physical agents in modulating pain. J Hand Ther 1997; 10: 110-21. [Crossref]

16. Thilander B, Rubio G, Pena L, de Mayorga C. Prevalence of temporomandibular dysfunction and its association with malocclusion in children and adolescents: an epidemiologic study related to specified stages of dental development. Angle Orthod 2002; 72: $146-54$.

17. Manfredini D, Stellini E, Gracco A, Lombardo L, Nardini LG, Siciliani G. Orthodontics is temporomandibular disorder-neutral. Angle Orthod 2016; 86: 649-54. [Crossref]

18. Motro PFK, Motro M, Oral K. Orthodontics and temporomandibular disorders. Are they related? Turkish J Orthod. 2015; 28: 71-6. [Crossref]

19. Tolevski Meshkova D, Di Giacomo P, Panti F, D'Urso A, Serritella E, Di Paolo C. Application of a systematic protocol in the treatment of TMDs with occlusal appliances: effectiveness and efficiency in a longitudinal retrospective study with medium-term follow-up. J Int Soc Prev Community Dent 2019; 9: 372-82. [Crossref]

20. de Resende CMBM, de Oliveira Medeiros FGL, de Figueiredo Rêgo CR, Bispo ASL, Barbosa GAS, de Almeida EO. Short-term effectiveness of conservative therapies in pain, quality of life, and sleep in patients with temporomandibular disorders: A randomized clinical trial. Cranio 2019; 15: 1-9. [Crossref]

21. Imai T, Okamoto T, Kaneko T, Umeda K, Yamamoto T, Nakamura S. et al. Long-term follow-up of clinical symptoms in TMD patients who underwent occlusal reconstruction by orthodontic treatment. Eur J Orthod 2000; 22: 61-7. [Crossref]

22. Vogl TJ, Lauer HC, Lehnert T, Naguib NN, Ottl P, Filmann N, et al. The value of MRI in patients with temporomandibular joint dysfunction: Correlation of MRI and clinical findings. Eur J Radiol 2016; 85: 714-9. [Crossref]

23. Massilla Mani F, Sivasubramanian SS. A study of temporomandibular joint osteoarthritis using computed tomographic imaging. Biomed J 2016; 39: 201-6. [Crossref]

24. Michelotti A, lodice G, Vollaro S, Steenks MH, Farella M. Evaluation of the short-term effectiveness of education versus an occlusal splint for the treatment of myofascial pain of the jaw muscles. J Am Dent Assoc 2012; 143: 47-53. [Crossref] 
25. Clark GT. A critical evaluation of orthopedic interocclusal appliance therapy: Design, theory, and overall effectiveness. J Am Dent Assoc 1984; 108: 359-68. [Crossref]

26. Conti PC, Santos CN, Kogawa EM, Castro Ferreira Conti AC, Araujo $\mathrm{CR}$. The treatment of painful temporomandibular joint clicking with oral splints: A randomized clinical trial. J Am Dent Assoc 2006; 137: 1108-14. [Crossref]

27. Al-Moraissi EA. Arthroscopy versus arthrocentesis in the management of internal derangement of the temporomandibular joint: A systematic review and meta-analysis. Int J Oral Maxillofac Surg 2015; 44: 104-12. [Crossref]

28. Le Bell Y, Jämsä T, Korri S, Niemi PM, Alanen P. Effect of artificial occlusal interferences depends on previous experience of temporomandibular disorders. Acta Odontol Scand 2002; 60: 219-22. [Crossref]

29. De Boever JA, Carlsson GE, Klineberg IJ. Need for occlusal therapy and prosthodontic treatment in the management of temporomandibular disorders. Part I. Occlusal interferences and occlusal adjustment. J Oral Rehabil 2000; 27: 367-79. [Crossref]

30. Packard RC. The relationship of neck injury and post-traumatic headache. Curr Pain Headache Rep 2002; 6: 301-7. [Crossref]

31. List $T$, Tegelberg A, Haraldson T, Isacsson $G$. Intra-articular morphine as analgesic in temporomandibular joint arthralgia/osteoarthritis. Pain 2001; 94: 275-82. [Crossref]

32. Rizzatti-Barbosa CM, Nogueira MT, de Andrade ED, Ambrosano GM, de Barbosa JR. Clinical evaluation of amitriptyline for the control of chronic pain caused by temporomandibular joint disorders. Cranio 2003; 2: 221-5. [Crossref]

33. Inagaki T, Miyaoka T, Shinno H, Horiguchi J, Matsuda S, Yoshikawa H. Treatment of temporomandibular pain with the selective serotonin reuptake inhibitor paroxetine. Prim Care Companion J Clin Psychiatry 2007; 9: 69-70. [Crossref]

34. Kreisberg MK. Tricyclic antidepressants: analgesic effect and indications in orofacial pain. J Craniomandib Disord 1988; 2: 171-87.

35. Dimitroulis $G$. Management of temporomandibular joint disorders: A surgeon's perspective. Aust Dent J 2018; 63: 79-90. [Crossref]

36. Horowitz L, Sarkin JM. Video display terminal operation: A potential risk in the etiology and maintenance of temporomandibular disorders. Cranio 1992; 10: 43-50. [Crossref]

37. Verkerk K, Luijsterburg PA, Heymans MW, Ronchetti I, Pool-Goudzwaard AL, Miedema HS, et al. Prognosis and course of pain in patients with chronic non-specific low back pain: A 1-year follow-up cohort study. Eur J Pain 2015; 19: 1101-10.v [Crossref]
38. Türp JC, Jokstad A, Motschall E, Schindler HJ, Windecker-Gétaz I, Ettlin DA. Is there a superiority of multimodal as opposed to simple therapy in patients with temporomandibular disorders? A qualitative systematic review of the literature. Clin Oral Implants Res 2007; 18: 138-50. [Crossref]

39. Rehm DD, Progiante PS, Pattussi MP, Pellizzer EP, Grossi PK, Grossi ML. Sleep Disorders in Patients with Temporomandibular Disorders (TMD) in an Adult Population- Based Cross-Sectional Survey in Southern Brazil. Int J Prosthodont 2020; 33: 9-13. [Crossref]

40. Veiga DM, Cunali R, Bonotto D, Cunali PA. Sleep quality in patients with temporomandibular disorder: A systematic review. Sleep Sci 2013; 6: 120-4.

41. Litt MD, Shafer DM, Kreutzer DL. Brief cognitive-behavioral treatment for TMD pain: Long-term outcomes and moderators of treatment. Pain 2010; 15: 110-6. [Crossref]

42. Voscopoulos C, Lema M. When does acute pain become chronic? $\mathrm{Br}$ J Anaest 2010; 105: 69-85. [Crossref]

43. Tate JJ, Milner C.E. Real-time kinematic, temporospatial, and kinetic biofeedback during gait retraining in patients: A systematic review. Phys Ther 2010; 90: 1123-34. [Crossref]

44. Shedden Mora MC, Weber D, Neff A, Rief W. Biofeedback-based cognitive-behavioral treatment compared with occlusal splint for temporomandibular disorder: A randomized controlled trial. Clin J Pain 2013; 29: 1057-65. [Crossref]

45. Tsai CM, Chou SL, Gale EN, McCall WD Jr. Human masticatory muscle activity and jaw position under experimental stress. J Oral Rehabil 2002; 29: 44-51. [Crossref]

46. Bae Y, Park Y. The Effect of Relaxation Exercises for the Masticator Muscles on Temporomandibular Joint Dysfunction (TMD). J Phys Ther Sci 2013; 25: 583-6. [Crossref]

47. Bär KJ, Wagner G, Koschke M, Boettger S, Boettger MK, Schlösser R, Sauer $\mathrm{H}$. Increased prefrontal activation during pain perception in major depression. Biol Psychiatry 2007; 62: 1281-7. [Crossref]

48. Sruthi VK, Jimsha SV, Srinivasan JM. Prevalence of Depression, Anxiety and Stress in Chronic Temporomandibular Joint Disorders Patients. J Depress Anxiety 2018; 7: 4.

49. Reissmann DR, John MT, Seedorf H, Doering S, Schierz O. Temporomandibular disorder pain is related to the general disposition to be anxious. J Oral Facial Pain Headache 2014; 28: 322-30. [Crossref]

50. Giannakopoulos NN, Keller L, Rammelsberg P, Kronmüller KT, Schmitter M. Anxiety and depression in patients with chronic temporomandibular pain and in controls. J Dent 2010; 38: 369-76. [Crossref] 